(R. Boivin, TFTR). In principle, each of these effects has been observed at TFTR through energetic protons $(p)$ and tritons $(t)$ created in the fusion reaction

$$
\mathrm{d}+\mathrm{d} \rightarrow \mathrm{p}(3 \mathrm{MeV})+\mathrm{t}(1.0 \mathrm{MeV})
$$

And in closely related work at JET (F. Marcus), 14.7-MeV protons from deuterium-helium-3 reactions were poorly confined with a 1.5 MA plasma current, but well confined with a 4.5 MA current unless sawtooth oscillations occurred. For deuterium-tritium plasmas, the crux of the issue will be quantitative: what fraction of the $\alpha$ particles will be so affected, and how often?

A conceptually related issue is whether the large population of $\alpha$-particles will themselves generate and drive formerly benign or unknown collective oscillations in the plasma that would again result in their 'self ejection'. Of the class of such instabilities that physicists theorize, none was more widely discussed than the $\alpha$-driven Alfvén mode $^{5-7}$. This instability should occur if a sufficient number of $\alpha$-particles have, along the direction of the magnetic field, a component of velocity greater than a critical velocity called the Alfvén velocity, $V_{\mathrm{A}}$ (D. Sigmar, MIT). (The tokamak's confining magnetic field includes a toroidal component and a smaller poloidal field created by the plasma current.)

The Alfvén velocity, which depends on the field strength $(B)$ and central ion density $\left(V_{\mathrm{A}}=B / \sqrt{4 \pi m_{\mathrm{i}} n_{\mathrm{i}}}\right)$, is around $0.8 \times 10^{9} \mathrm{~cm}$ $\mathrm{s}^{-1}$ (with a field of 50 kilogauss and central ion density of $6 \times 10^{13} \mathrm{~cm}^{-3}$ ). The initial velocity of the $3.5-\mathrm{MeV} \alpha$-particles is $1.3 \times 10^{9}$ $\mathrm{cm} \mathrm{s}^{-1}$, and about 40 per cent will have a 'parallel' velocity component exceeding the Alfvén velocity.

But it is also becoming apparent that two other conditions need to be satisfied if the Alfvén mode is to be excited. First, the number density of $\alpha$-particles at the centre must not be less than about 0.3 per cent of the electron density. And second, the $\alpha$-particles' gas-kinetic pressure must exceed 0.003 times the magnetic pressure they experience ( $B^{2} / 8 \pi$, about 100 atmospheres).

Simulations of TFTR deuterium-tritium plasmas (R. Bundy) find all three thresholds likely to be exceeded. But recent " $\alpha$-simulation experiments" (K. Wong) on TFTR using energetic neutral beams and deuterium plasmas indicate that the pressure threshold may be larger by about an order of magnitude $^{\text {? }}$. If so, then TFTR deuterium-tritium plasmas may be spared this particular $\alpha$-particle loss channel.

The best method of injecting tritium into TFTR is also of pressing importance. The most frequently discussed methods are injecting it either with high-power heating beams, or firing in tiny frozen tritium pellets. Among other compelling arguments for using pellets (each less than $0.01 \mathrm{~g}$ in mass, equivalent to 100 curies of radioactivity), one is that it will tend to place the tritium mostly on the tokamak axis where it can be more effectively burned (D. Mikkelsen, TFTR). Another not inconsequential benefit is that pellet injection reduces by an order of magnitude the total amount of tritrium injected into the machine (D. Meade, TFTR).

Making maximum use of the limited number of deuterium-tritium discharges is another concern (J. Strachan, TFTR); only 1,000 or so are feasible, whereas TFTR has already had about 50,000 hydrogen, deuterium and helium discharges. For example, should new diagnostic methods be developed that in principle could, with some spatial and temporal resolution, measure aspects of the $\alpha$-particles' energy distribution? Although highly desirable, the means to measure these parameters are at present untested, although serious efforts to do just that are underway (P. Woskov, MIT). And even if not immediately applicable to TFTR, they might prove invaluable for the next generation of experiments.

An approach that addresses the immediate needs of TFTR is to place greater emphasis on proven methods that could measure the escaping $\alpha$-particles (as with tritons as shown in the figure). Largely in combination with the $\alpha$-particle birth profile (from 14.1-MeV neutron imaging), such measurements should reveal a clear picture of the overall $\alpha$-particle transport.

Furthermore, it will be essential to establish experimentally for each diagnostic technique - such as those used to measure the ion-temperature profile or the plasma impurity content - its ability to operate reliably in the intense neutron and $\gamma$-ray fluxes resulting from the fusion neutrons. None of the tokamak diagnostic techniques has yet operated in such a severe environment, so that there is concern that, despite careful shielding and other precautions, some measurements might give false or unintelligible signals owing to the background radiations.

Finally, assuming that most of the $\alpha$-particles do deposit their energy in the plasma, what is to be done with the thermalized $\mathrm{He}^{2+}$ 'ash' which would certainly dilute the deuterium-tritium fuel and so extinguish hightemperature ignition? One proposed solution (G. Miley, Univ. Illinois) is that suitable plasma instabilities should be periodically triggered so as to expel the ash. However, from this and previous considerations it is clear that a fine line has to be walked: on the one hand energetic $\alpha$-particles must deposit their energy in the plasma; on the other, having done so, they must be transported out. TFTR should give us our very first glimpse of this delicate balance.

Richard D. Petrasso is at the Plasma Fusion Center, Massachusetts Institute of Technology, Cambridge, Massachusetts 02139, USA.

1. Meade, D. et al. IAEA 1990, W. D. C. (Proc. to be published). 2. Keilhacker, M. et al. Phys. Fluids 2, 1291 (1990).

3. Marmar, E. et al. Nucl. Fus. 22, 1567 (1982).

4. Marmar, E. et al. Nucl. Fus. 22, 1567 (1982).

5. Fu, G. Y. \& Van Dam, J. Phys. Fluids B1, 1949 (1989).

6. Sigmar, D. S., Hsu, C., White, R. \& Cheng, C. Z. MiT Piasma Fusion Center, Rep. No. PFC/JA-89-58 (1989)

7. Wong, K. L. et al. Phys. Rev. Lett. 66, 1874-1877 (1991).

\section{Dead Heat}

LAST week Daedalus proposed a novel sound-absorber. It is a rubber plasticized with high-pressure gas, so that the contractile tension in the rubber matrix is exactly balanced by the pressure of the occluded gas. Over a wide density-range it has no preferred volume and zero elasticity. So sound traverses it with zero velocity, and never gets anywhere.

Both as a perfect sound-insulator, and as a completely 'dead' vibration-damper, the new polymer/gas composite will find innumerable applications. But Daedalus now points out that heat is also a form of sound; it is propagated by thermal phonons. A gas-swollen polymer balanced to have zero phonon-velocity should be a perfect heat-insulator.

At last the bulkiness and inconvenience of conventional lagging will be overcome. Even in thin sheet form, DREADCO's new "Dead Heat" polymer will totally insulate refrigerators and ovens, hot-water tanks and pipes, walls and windows, floors and ceilings, and all other surfaces through which heat leaks wastefully away. Offices will be fully heated by their content of staff and computers, frozen foods and hot drinks will be sold on open shelves in Dead Heat cartons, and a new market may open up for well-wrapped parcels of industrial waste heat. And on the human scale, the unstated modern tension between fashion and domestic heating will at last be resolved. Respectable victorians, with many layers of woollen underwear, could tolerate houses at $10{ }^{\circ} \mathrm{C}$. We demand a wasteful $20{ }^{\circ} \mathrm{C}$ in order to show off our bodily elegance under a minimum thickness of tight-fitting clothing. But tailored in DREADCO's Dead Heat fabrics, even the thinnest jeans, most figure-hugging gowns or negligible negligees will be as warm and comfortable as arctic survival-gear.

They may feel a bit like it, too. The new fabrics, with their complete absorption of movement and vibration, will insulate the wearer from the innumerable small touches and contacts which make up kinetic awareness. The resulting sense of isolation and numbness could be quite disturbing, especially in clothes like gloves, boots and underpants. The material may have to be slightly unbalanced, giving it a slight vibration-velocity.

Such a material would also make an intriguing thermal delay-line. A high temperature applied to one side of it would emerge from the other side maybe hours later. This could be very useful. A housewall of the product, for example, could be formulated to have a twelve-hour delay. More economical even than a total thermal insulator, it would retard the warmth of the day till night-time, while allowing the cool of midnight into the premises around noon.

David Jones 